\title{
Emoción expresada: Introducción al concepto, evaluación e implicaciones pronósticas y terapéuticas
}

\section{Carmen Vizcarro \\ Jorge Arévalo}

Universidad Autónoma de Madrid

\section{INTRODUCCION*}

Junto a importantes esfuerzos en el estudio de la etiología de la esquizofrenia, especialmente en las áreas relacionadas con sus bases biológicas (fundamentalmente características bioquímicas y neurofisiológicas), se está insistiendo últimamente en el fenómeno de causación multifactorial que supone esta patología. Efectivamente, parece evidente que, a pesar de reconocer el papel que desempeñan los factores orgánicos en la aparición de esta patología, éstos no son suficientes, en la gran maỹoría de los casos, para producirla, en ausencia de otras circunstancias que, uniendo su efecto al de aquéllos, determinan la aparición de desviaciones severas en diversas áreas del funcionamiento del individuo. Se apela aquí a los efectos de diversos factores estresantes, más o menos normativos o a la influencia de diversos factores ecológicos, sociales o culturales. Esta compleja interacción de factores queda reflejada en modelos explicativos de segundo orden como el de vulnerabilidad (Zubin y Spring, 1977; Zubin y Steinhauer, 1981; Vizcarro, Botella y Ruiz Vargas, 1982; Vizcarro, en preparación).

Estos factores externos cobran una gran relevancia, cara a la intervención psicológica, especialmente en un momento en que la atención a estos pacientes tiende a restringir al máximo los períodos de hospitalización, maximizando, en cambio, la prevención primaria y secundaria. Dentro del área de la rehabilitación, igualmente, se ha demostrado que la prevención de futuros episodios resulta más eficaz al recurrir conjuntamente a la intervención simultánea a diversos niveles, incluyendo tratamientos tanto farmacológicos como socioambientales (ver, por ejemplo, May, Tuma y Dixon, 1981).

Más en concreto, diversas dimensiones de la vida familiar han llamado la atención tanto de clínicos como de investigadores, habiéndoseles incluso atribuido un papel causal en el desarrollo de estos trastornos (Bateson, Jackson, Haley y Weakland, 1956; Lids, 1958; Wynne, Rycjoff, Day y Hirsch, 1958). Actualmente, el cuerpo de investigaciones sobre la emoción 
expresada (EE desde ahora) por familiares de los sujetos esquizofrénicos, constituye, probablemente, uno de los intentos más serios por operativizar estas variables y por estudiar sus efectos de un modo empírico. Efectivamente, se encontró inicialmente que el pronóstico de pacientes esquizofrénicos al ser dados de alta guardaba una estrecha relación con el contexto que les recibía, especificando una serie de características de las interacciones sociales entre el paciente y sus familiares $\mathrm{y} / \mathrm{u}$ otras personas del entorno que parecían incidir sobre la evolución positiva o negativa de los trastornos (Brown, Monck, Carstairs y Wing, 1962).

\section{El concepto de emoción expresada y su evaluación}

El concepto de EE ha surgido de modo pragmático para designar algunos aspectos de la conducta emocional que dentro de un núcleo familiar se manifiestan hacia sus miembros enfermos o que presentan trastornos de conducta. La noción es puramente pragmática en cuanto que sólo engloba aquellos aspectos de la conducta emocional de los familiares o personas con las que convive un paciente y que ejercen una influencia significativa sobre el curso de los trastornos y, por todo ello, resultan útiles de cara a un pronóstico. Como es lógico, la observación de estas relaciones nos permite igualmente, y estos son los últimos desarrollos del concepto, establecer ciertas indicaciones terapéuticas.

En cualquier caso, es importante tener presente que no se pretende llevar a cabo una evaluación exhaustiva de los intercambios emocionales que se producen entre familiares. Como ya queda dicho, sólo se incluyen aque: llas variables que, empíricamente, han demostrado tener un valor pronóstico.

Es necesario, igualmente, hacer un esfuerzo para desligar este concepto de los enfoques familiares tradicionales. En efecto, se observa en este ámbito una decidida voluntad por evitar cualquier tipo de especulación teórica y, por el contrario, por ajustarse del modo más estricto posible a los datos empíricos de los que se dispone. En efecto, de los intentos iniciales de Brown y colaboradores por aislar dimensiones de la vida familiar que resultaran relevantes para el curso de los trastornos esquizofrénicos, y a partir de un fondo inicial de cientos de características, se fue depurando un pequeño conjunto de dimensiones con capacidad predictiva. Estas dimensiones han sido agrupadas bajo la etiqueta de «emoción expresada» y sólo posteriormente se está intentando estudiar, a través de sus correlatos a distintos niveles, la naturaleza de este conjunto de medidas. En efecto, consecuentemente con la naturaleza de los datos iniciales, nunca se teorizó con lo que estas variables podrían implicar dentro de la dinámica familiar ni se hicieron especulaciones sobre el posible valor eriológico de las mismas en los trastornos esquizofrénicos; los datos demostraban, simplemente, su influencia en el curso de los trastornos. Posteriormente se han llevado a cabo numerosos estudios (y podríamos decir que éste sigue siendo su objetivo prioritario en la investigación en esta área) con objeto de determinar, más exactamente, la naturaleza de lo que se está midiendo. De este modo, por ejemplo, se ha obtenido evidencia de que tal vez no se trate de un concepto unitario, dado que las correlaciones de sus componentes con diversas variables muestran diferencias importantes. Incluso, se han reinterpretado estas medidas desde la perspectiva del control social; de este modo, las variables que se registran no serían tomadas como indicadores de la atmósfera emocional de un hogar, sino como medidas de con- 
trol social que los familiares ponen en juego para intentar conseguir del paciente una conducta más acorde con las normas sociales (ver epígrafes 2 y 3 sobre estos puntos). Así pues, podemos considerar que la etiqueta es en cierto modo provisional y, hasta podríamos decir, equívoca, en la medida en que puede no reflejar con claridad lo que realmente se está midiendo. No obstante, hasta el momento se ha acumulado en torno a esta denominación un importante fondo bibliográfico sobre la materia, por lo que pensamos que es preferible, aún con las limitaciones señaladas, conservarla y presentarla aquí con una traducción literal de la misma. Por otra parte, el enfoque empírico que, como hemos comentado, preside los desarrollos en esta área constituye una garantía de que, si no el término, al menos el concepto pueda ir experimentando los reajustes necesarios.

En cuanto a su contenido específico, la EE se refiere a aspectos emocionales de la conducta de los miembros que componen el entorno familiar de los pacientes, que son observados directamente en la entrevista o bien de los que informa el propio familiar y que tienen lugar en situaciones de la vida cotidiana. A partir de estas observaciones se clasifica a los familiares en alta o baja EE y, sobre la base de estas valoraciones correspondientes a los individuos, a la familia como un todo. En el momento. actual, puede decirse que el constructo «emoción expresada» consta básicamente de dos componentes: implicación emocional excesiva y actitud crítica, que puede llegar a conceptuarse como hostilidad, por parte de los familiares que van a recibir al paciente a su regreso a la comunidad.

En relación con la metodología para recoger dicha información, después de los trabajos pioneros ya citados de Brown y sus colaboradores para identificar los componentes del clima familiar que pueden estar contribuyendo a las reiteradas recaídas de los pacientes esquizofrénicos, se desarrolló una entrevista semiestructurada con opciones de registro de información de tipo escalar, a la que se denominó Camberwell Family Interview o CFI (Brown y Rutter, 1966; Rutter y Brown, 1966). Dicha entrevista recoge tanto información objetiva como subjetiva. La primera está formada por acontecimientos observables como la distribución del tiempo por parte del paciente y familiares, la frecuencia e intensidad de su interacción mutua o el reparto de responsabilidades de las tareas domésticas. La información subjetiva se refiere a sentimientos, tanto de naturaleza positiva como negativa y referidos al pasado o al presente y a su expresión en forma de conducta. Estos se deducen del contenido del lenguaje, así como de aspectos paralinguísticos como el tono de voz y el lenguaje no verbal.

La duración inicial de la entrevista era de tres o cuatro horas, siendo abreviada posteriormente hasta aproximadamente hora y media sin variaciones sustancialles en la información obtenida (ver Vaughn y Leff, 1976a). Desde el punto de vista de su contenido, se explora el inicio del episodio actual, el grado en que éste ha afectado a la vida familiar en aspectos como la realización de las tareas domésticas, el grado de tensión en el hogar y similares; asimismo, se explora el grado de control que el familiar cree que el paciente tiene sobre la situación, o su satisfacción en la misma (puede encontrarse información más detallada en Leff y Vaughn, 1985). Aunque los acontecimientos constituyen, por sí mismos, una fuente útil de información, el aspecto más relevante desde el punto de vista de la EE lo constituyen los sentimientos que los familiares muestran hacia el paciente a lo largo de la entrevista, así como las conductas de las que informan con respecto al mismo en su vida cotidiana. Como señalan Rutter y Brown (1966): «el contenido de lo que se dice es tenido en cuenta, pero se presta 
más atención al cómo se dicen las cosas. Se espera que los entrevistadores reconozcan emociones observando diferencias en la velocidad, el tono y la intensidad del habla. En menor medida se consideran la expresión y los gestos» (pág. 45).

Estas entrevistas son grabadas y posteriormente valoradas en las cinco escalas que orginalmente constituían el constructo de emoción expresada: criticismo, hostilidad, implicación emocional excesiva, calor y comentarios positivos. En efecto, a pesar de que sólo las tres primeras escalas han demostrado tener validez pronóstica, se siguen conservando las cinco con objeto de recoger información que puede ser de interés para la planificación terapéutica. A continuación describiremos brevemente cada una de estas escalas.

Comentarios críticos (CC): Con esta denominación se hace referencia a la suma total de comentarios críticos realizados por el familiar acerca del paciente a lo largo de la entrevista. Para que un comentario sea considerado crítico, se considera el contenido del mismo, así como el tono de voz con que se hace. El contenido debe ser una observación sin ambigüedad de resentimiento, disgusto o deșaprobación hacia el paciente o su comportamiento, tal como el siguiente realizado por el padre de un paciente de veintiocho años de la muestra española: «se está comportando como un auténtico guarro, no se molesta ni en recoger un cenicero». Si la decisión de valorar esta categoría se toma en base al tono de voz, se deben tener en cuenta la intensidad, la velocidad y la inflexión, de la comunicación, de forma que una observación de contenido inocuo puede llegar a ser considerada crítica si los parámetros mencionados así lo aconsejan. En la medida en que la puntuación obtenida en esta escala exige un recuento del número total de comentarios críticos que el familiar ha emitido a lo largo de la entrevista, un aspecto esencial lo constituye la distinción entre ocurrencias discretas de comentarios críticos. En este sentido, se definen como ocurrencias distintas aquellos comentarios que se refieren a diferente tema, o bien que, auh perteneciendo al mismo aspecto del paciente o su conducta, van separados por preguntas del entrevistador.

Hostilidad $(H)$ : Es un tipo más extremo de comentario crítico, cuya valoración en contraste con lo ya descrito, se basa exclusivamente en el contenido. Mientras que los comentarios críticos se refieren al malestar expresado respecto a conductas y/o situaciones específicas, la hostilidad implica una impresión negativa generalizada o un rechazo manifiesto del paciente como persona y no sólo de su conducta. Por ejemplo: «Nunca saluda, ni dice buenas tardes, ba sido siempre un despistado para sus hijos y para todo, nunca ba becho nada bien, o bien una madre que dice: ese no vuelve a entrar en mi casa». La escala de hostilidad, a diferencia de la anterior, no está constituida por un recuento total del númeło de comentarios de esta naturaleza, sino que es una escala global de cuatro puntos $(0=$ ausente, $1=$ presente como generalización, 2 =presente como rechazo y 3 = presente como generalización y rechazo simultáneamente), basada en la entrevista como un todo. Así, si a lo largo de una entrevista pueden encontrarse comentarios que impliquen generalización, pero no rechazo, la valoración final de esta escala será de 1 , con independencia del número concreto de comentarios de esta naturaleza.

Implicación emocional excesiva (IEE): Esta escala tiene una composición compleja. Por una parte, refleja una respuesta emocional exagerada y desproporcionada del familiar ante el trastorno del paciente (por ejemplo, lloros muy frecuentes durante la entrevista o comentarios como «estoy perdiendo la vida... Llevamos once años que no han sido vivir»). Por 
otra parte, incluye igualmente una conducta exageradamente sobreprotectora hacia el paciente o que implica un sacrificio excesivo para el familiar. Por ejemplo, en rẹlación con el alquiler de una casa en la playa, el padre de un paciente de treinta y un años comentó: "yo no puedo pagar ese alquiler, pero me sacrifico y lo hago, lo hago por él». Finalmente, se valoran igualmente en esta escala los intentos, por parte del familiar, de ejercer un control excesivo sobre la conducta del paciente (prepararle horarios a los que se debe ajustar, o seleccionar sus amigos). Así, pues, como vemos, la IEE se refiere a tipos de emociones muy diversos. Puede existir cierta tendencia, antes de familiarizarse con este concepto, a identificarlo con el más conocido, en el área de la interacción familiar, de sobreprotección. Es necesario señalar, a este respecto, que si bien ambos conceptos coinciden en cierta medida, no pueden considerarse en modo alguno equivalentes, ya que la IEE comprende también otras manifestaciones emocionales, es decir, es más amplio.

Al igual que la hostilidad, la implicación emocional excesiva es una escala global en la que las valoraciones se realizan sobre el conjunto de la entrevista, constituyendo un juicio complejo que se refleja en una escala ordinal de 0 a 5 ( 0 = ausencia, 5 = acusada). Naturalmente, para llevar a cabo esta valoración es necesario tener en perspectiva la relación que une al paciente y familiar (por ejemplo, entre los miembros de una pareja serían más tolerables y esperables conductas que pudieran considerarse sobreprotectoras que entre padres e hijos), así como la edad y nivel de funcionamiento del paciente.

Calor $(C)$ : Por tal se conceptualizan las manifestaciones de simpatía, afecto, e interés, así como la presencia de empatía y comprensión hacia la persona del paciente («pobrecito, lo que ha tenido que sufrir» o «me da mucha pena verlo en el hospital»). La espontaneidad de este tipo de manifestaciones es un aspecto importante para su valoración, en la que son tenidos en cuenta el contenido del lenguaje y, especialmente, el tono de voz. Constituye, como la anterior, una escala global que va de 0 a 5 puntos.

Comentarios positivos $(C P)$ : Son observaciones, como su propio nombre indica, positivas, en las que se expresa una clara aprobación, valoración o aprecio del paciente o de su conducta ( no es porque yo lo diga, pero es un chico estupendo»), valorándose exclusivamente sobre la base del contenido. Como en el caso de los comentarios críticos, esta escala supone un recuento numérico de los comentarios positivos hechos a lo largo de la entrevista.

Como ya hemos señalado, sólo las tres primeras escalas se incluyen en el índice de emoción expresada. La definición de dicho índice ha experimentado sucesivas modificaciones con objeto de depurar su capacidad predictiva (Brown, Birley y Wing, 1972; Leff y Vaughn, 1976a; Vaughn, Snyder, Jones et al., 1984). En los estudios más recientes se han fijado los siguientes puntos de corte, calificando a una familia como alta en EE cuando uno al menos de sus miembros recibe una de las siguientes valoraciones: 6 o más comentarios críticos; una puntuación de 3 o más en implicación emocional excesiva; presencia de hostilidad, en cualquiera de sus alternativas, o cualquier combinación de las anteriores. Es importante resaltar que estas escalas son unidimensionales y se valoran con total independencia unas de otras. Señalemos, no obstante, que resulta extraño encontrar una valoración de hostilidad que no vaya acompañada de un alto número de comentarios críticos (ver, para una información más detallada, Leff y Vaughn, 1985).

El interés por la fiabilidad de estas medidas se ha centrado, hasta el 
momento, exclusivamente en el acuerdo interjueces. Este interés en la objetividad de la medida parece lógico, al menos inicialmente, dada la naturaleza de los conceptos que se pretende medir, así como la definición de los mismos, que sin duda puede considerarse subjeriva. Podemos decir, a pesar de ello, que ésta es elevada. Brown, Birley y Wing informan de un acuerdo interjueces entre 0,80 y 0,90 en las distintas escalas de la CFI. Asimismo, Vaughn y Leff (1976a) encontraron una fiabilidad global, de 0,86 en comentarios críticos. Los autores de este trabajo han obtenido una fiabilidad entre 1 (coeficiente phi, para la escala de Hostilidad) y 0,81 (rho de Spearman, para la valoración de calor) con un coeficiente de 0,86 (coeficiente phi) para la valoración del nivel global de emoción expresada. Es necesario advertir, no obstante, que estos niveles de fiabilidad exigen un costoso entrenamiento que proporcionan directamente algunos de los autores a los que nos hemos referido.

\section{Vàlidez y utilidad del concepto de emoción expresada}

Simultáneamente a la depuración del procedimiento de medida (Brown y Rutter, 1966; Rutter y Brown, 1966), se fueron replicando los hallazgos a los que hemos aludido anteriormente, así como poniendo de relieve la interacción de estos factores con otros de conocido valor pronóstico, como los acontecimientos estresantes o el ajuste premórbido, así comó especificando la relación de distintos componentes del constructo con el pronóstico. Igualmente, se aisló la contribución al pronóstico de otras variables relevantes, como la desorganización conductual, la historia laboral o es estado clínico del paciente, diversas características demográficas, la acción de la medicación de mantenimiento, la actitud del paciente frente a su propio ingreso o el nivel de aislamiento social de los otros significativos. (Brown, Birley y Wing, 1972; Vaughn y Leff, 1976a). Estos últimos autores concluyen, como resultado de estos estudios, que la EE constituye el mejor predictor individual de recaída en sujetos esquizofrénicos (Vaughn y Leff, 1976b), datos éstos que se han visto confirmados en estudios posteriores (véase Vaughn, Snyder, Jones, Freeman y Falloon; 1984). En efecto, se ha comprobado que existe una fuerte asociación de los componentes de la EE con las recaídas tanto en un seguimiento a los nueve meses (Brown et al., 1962; Brown, Birley y Wing, 1972; Vaughn y Leff, 1976a) como a los dos años (Leff y Vaughn, 1981). Esto es igualmente cierto para las muestras inglesas originales como para muestras americanas (Vaughn, Snyder, Freeman, et al., 1982; Vaughn, Snyder, Jones et al, 1984). Como puede apreciarse en Vaughn y Leff (1976a) los datos obtenidos en estos estudios, de una magnitud muy similar en todos ellos, no dejan lugar a dudas sobre el valor pronóstico de estas variables.

En estos mismos estudios se ha comprobado, no obstante, que existen algunas variables que modulan esta relación de la EE con el pronóstico. Así, por ejemplo, la variable sexo, como la edad o la relación del paciente con sus familiares (si es hijo o cónyuge) parece matizar las relaciones observadas entre el nivel de EE y las recaídas (Brown, Birley y Wing, 1972; Vaughn y Leff, 1976a; Vaughn et al., 1984). Existen, por otra parte, algunos factores, que, dadas estas circunstancias, pueden actuar como protectores: en concreto, la ingestión regular de medicación de mantenimiento y una restricción del contacto personal con los miembros de la familia que muestran un alto nivel de EE a menos de 35 horas semanales. Asimismo, resulta lógico pensar que un alto nivel de EE puede ser una fuente de es- 
trés mucho más importante si la vulnerabilidad del paciente es mayor, si su red social es más estrecha y menos cohesionada (ejerciendo, por lo tanto, en menor medida su efecto protector), o, finalmente, si el paciente dispone de estrategias cognitivas que le permiten minimizar el efecto desfavorable de estas manifestaciones negativas de emoción. Faltan, sin embargo, estudios sobre la influencia de estas últimas variables. En la U.A.M. se están llevando a cabo algunos de ellos.

A pesar del resultado de los estudios antes citados, que parecían descartar la posibilidad de que el valor pronóstico de la EE pudiese ser considerado como un artefacto, a través de su relación con otras variables de conocido valor pronóstico, la relación de la EE con otros índices pronósticos ha sido estudiada específicamente por Miklowitz, Goldstein y Falloon (1983). Al igual que en los estudios anteriores, estos autores no encontraron inicialmente diferencias en sintomatología o ajuste premórbido entre los pacientes de alta y baja EE. Ahora bien, cuando el grupo de alta EE fue analizado en subtipos (alta EE debida a IEE o a un alto nivel de criticismo), se detectaron algunas diferencias sugerentes. En efecto, los pacientes procedentes de hogares con IEE prsentaban un peor ajuste premórbido y más sintomatología residual en el momento del alta que los de familias con un alto nivel de criticismo o de baja EE, grupos éstos que no se diferenciaban entre sí en estas dos variables. Como es bien sabido, ambas pueden ser consideradas como indicadores de buen pronóstico. Naturalmente, estos resultados sugieren que, en el caso de los pacientes con alto criticismo en sus hogares, la EE es sumamente relevante, por sí misma, para predecir las recaídas. En cambio, en los pacientes con familias con IEE esta dimensión covaría con otras características del paciente que predicen un ajuste deficiente. Cabe por ello cuestionarse si la EE tiene el mismo valor pronóstico, con independencia de dichas variables de los pacientes o si, en definitiva, una IEE puede ser un estilo de adaptación de la familia ante determinadas características del paciente con un bajo nivel de funcionamiento ( $o$, lo que es más probable, que ambas interactúen).

Estas observaciones han sido incorporadas a una teoría según la cual los sujetos esquizofrénicos presentarían una hipersensibilidad a su entorno social; cuando sus interacciones con un medio que presenta un alto nivel de EE son intensas, el resultado sería un nivel de activación extremadamente elevado y muy prolongado en el tiempo, lo que daría lugar, ya sea aisladamente o en conjunto con la ocurrencia de otros factores estresantes, a una reactivación de la sintomatología. Es relevante, a este respecto, el trabajo de Leff y Vaughn (1980). Estos autores estudiaron la interacción entre dos tipos de estrés (continuado, o EE y puntual, o acontecimientos vitales) en dos trastornos diferentes, depresión y esquizofrenia. Sus resultados indicaron interacciones de signo opuesto entre estos dos tipos de estrés en esquizofrénicos y depresivos: en los primeros era mayor el número de acontecimientos estresantes entre los de familias de baja EE que en los de alta $\mathrm{EE}$; en los depresivos, por el contrario, los que vivían con familiares de alto criticismo mostraron una alta tasa de ocurrencias de acontecimientos estresantes, comparados con los que procedían de familias con un bajo nivel de EE. Es decir, mientras que en la aparición de los trastornos esquizof rénicos parecía bastar la presencia de uno de los dos tipos de estrés, en los depresivos tenían que confluir dos de ellos para causar una depresión que los autores denominan «reactiva» (el otro grupo de depresivos es considerado por los autores como «depresión no precipitada» y, en este sentido, más próximo a la endógena). Por otra parte, Leff, Kuipers, Berkowitz, et al. (1983) encontraron que la toma regular de la 
medicación protege a los pacientes esquizofrénicos tanto del estrés agudo procedente de acontecimientos vitales como del más estable o "crónico» asociado al alto nivel de EE de sus familiares; no obstante, este efecto protector disminuye si ambos tipos de estrés se dan simultáneamente.

Los estudios en esta área se han centrado fundamentalmente, hasta el presente, como estamos viendo, en la influencia de la EE en el curso de los trastornos, sin pronunciarse sobre su papel etiológico. Es necesario enfatizar, por otra parte, el modo estricto como los autores que han trabajado en esta área han rehuido cualquier tipo de especulación no avalada por los datos. Efectivamente, en la mayor parte de estos estudios no resulta posible distinguir si un nivel elevado de EE por los familiares desempeña un papel causal en el pronóstico de los pacientes (y mucho menos, de los trastornos que presentan) o si, por el contrario, dicho nivel de EE constituye un efecto de la existencia de un miembro perturbado en la familia. Hemos visto, en el estudio de Miklowitz, Goldstein y Falloon, alguna evidencia que permitiría apoyar esta suposición. Existen, no obstante, algunos datos positivos acerca de la relación causal entre la EE y el pronóstico. Así, los estudios que recurren a un diseño experimental, modificando algunas de las variables predictoras a las que hemos aludido (IEE o Criticismo) parecen apuntar en este sentido (ver, por ejemplo, Leff, Kuipers, Berkowitz et al., 1982 y epígrafe 4).

No obstante, estos estudios no aportan luz sobre el posible papel de la EE en el desarrollo inicial de estos trastornos. Consecuentemente, los autores eluden cuidadosamente toda suposición al respecto. Sin embargo, se han llevado a cabo últimamente algunos estudios longitudinales que parecen apuntar a la influencia del estilo afectivo de los padres en el desarrollo de cuadros patológicos comprendidos en el «espectro de la esquizofrenia» en sus hijos adolescentes (Doane, West, Goldstein et al., 1981). Es lógico pensar, no obstante, que la causalidad entre estos hechos no sea lineal (ya sea en un sentido o en otro), sino que se produzca un sistema interactivo que se retroalimente.

Otro conjunto de estudios se refieren, más bien, a las implicaciones prácticas de los hallazgos ofrecidos por el primer grupo de observaciones de cara a la planificación terapéutica. En otras palabras, exploran la posibilidad de actuar sobre el pronóstico modificando las relaciones observadas. Como ya hemos señalado, estos estuḍios, además de su evidente interés práctico, arrojan cierta luz sobre el posible valor causal de la $\mathrm{EE}$ en el pronóstico de la esquizofrenia. Así, Brown, Birley y Wing (1972) apuntan una serie de sugerencias prácticas que deberán guiar la intervención clínica con estos pacientes:

- Asegurar la toma regular de la medicación de mantenimiento,

- Reducción del número de horas de interacción directa entre el paciente y sus familiares, en el caso de pacientes con un alto nivel de EE,

- En otro orden de cosas, favorecer el ajuste laboral de un paciente.

Es necesario aclarar que, simultáneamente a la aplicación de estas medidas de sentido común, se han desarrollado recientemente estrategias de intervención con objeto de modificar el nivel de EE (ver epígrafe 4).

Diversos trabajos posteriores se han planteado el estudio empírico de la validez de estas conclusiones lógicas. Así, Leff y Vaughn (1981) encontraron que el efecto profiláctico de la medicación de mantenimiento no resultaba evidente en un seguimiento a los dos años para pacientes procedentes de familias con un alto nivel de EE y sí, en cambio, en pacientes sometidos a un bajo nivel de EE, en congruencia con lo que se desprende 
de las conclusiones de Brown, Birley y Wing (1972). Vaughn et al. (1984), en cambio, encontraron, con una muestra norteaméricana, que este efecto protector de la medicación sólo se producía en las familias de alta EE cuando el paciente mantenía un contacto directo inferior a las 35 horas semanales. Puede, por lo tanto, decirse, que la toma regular de medicación, así como la reducción de la interacción directa del paciente con sus familiares, constituyen factores protectores contra las recaídas cuando el medio familiar es alto en EE (Vaughn y Leff, 1976a; Vaughn et al. 1984). No obstante, estos factores han revelado igualmente un efecto beneficioso para evitar las recaídas de pacientes procedentes de familias de baja EE en un seguimientos a más largo plazo, mientras que no lo serían para aquellos con alta EE (Leff y Vaughn, 1981). En cualquier caso, puede merecer la pena evaluar estos factores sociales en aquellos casos en que la medicación no parece surtir los efectos beneficiosos esperados.

En cuanto a los programas dirigidos a modificar estas variables, podemos señalar que Leff, Kuipers, Berkowitz et al. (1982) comprobaron, en un seguimiento a los nueve meses, que el grupo de pacientes procedentes de medios con un alto nivel de EE mostraba un pronóstico más favorable si era sometido a tratamiento psicosociales con el objetivo fundamental de reducir la expresión de emociones negativas por parte de sus familiares, así como la interacción directa entre éstos y los pacientes. En el mismo snetido, Leff, Kuipers, Berkowitz et al. (1984) comprobaron los efectos benéficos de un tratamiento múltiple que incluía medicación preventiva y tratamiento psicosocial de las familias con un alto nivel de $\mathrm{EE}$, tanto en un seguimiento a los nueve meses como a los dos años (véase, para más información, el epígrafe 4).

\section{Tendencias futuras en la investigación sobre el constructo de emoción expresada}

De todo lo anteriormente dicho se desprende el interés práctico de este tipo de estudios. Tal vez menos evidentes, por posponerse a este interés práctico, sin embargo, surgen algunas cuestiones de carácter conceptual y metodológico planteadas por ellos y que resulta de fundamental importancia resolver. En primer lugar, ¿qué se está midiendo realmente? Esta cuestión básica se relaciona directamente, por otra parte, con una serie de cuestiones metodológicas que mencionaremos a continuación. Si, según parece, son actitudes lo que se está midiendo, es posible pensar que éstas puedan reflejarse en conductas concretas observables en la interacción familiar. En consecuencia, es posible, igualmente, plantearse su medida por medios tal vez más fácilmente objetivables que los actualmente utilizados. A su'vez, esto es posible ya sea directamente, tal y como se manifiestan en la interacción personal, o bien indirectamente, por sus efectos sobre el paciente.

Algunos estudios han abordado estas cuestiones con un objetivo doble. Por una parte, validar las medidas actuales, aportando información sobre los procesos en juego y, tal vez, como resultado de ello, depurar estrategias de medida más fácilmente objetivables (y, por lo tanto, más económicas) y con mejores perspectivas de incorporación a la práctica clínica cotidiana. Así, Tarrier, Vaughn, Lader y Leff (1979) y Sturgeon, Kuipers, Berkowitz et al. (1981) han encontrado, recurriendo a registros psicofisiológicos, datos que sugieren que los familiares con un alto nivel de EE producen un efecto activador sobre los pacientes que impide la habituación de respuestas electrodérmicas registradas en presencia de aquellos. Por el 
contrario, estos estudios parecen indicar que los familiares con un bajo nivel de EE favorecen la habituación de esta respuesta. Naturalmente, es lógico pensar que este efecto activador puede interferir con los esfuerzos adaptativos de los pacientes, especialmente en momentos en que coinciden con el impacto de otras fuentes de estrés, y aumentando, en definitiva, las dificultades de adaptación que éstos experimentan.

Naturalmente, las conductas mencionadas constituyen correlatos de la EE, lo que amplía nuestro conocimiento sobre este concepto. En esta línea, Vaughn y Leff (1981) citan, junto a estos efectos psicofisiológicos de la respuesta emocional de los familiares con alta $\mathrm{EE}$, su nivel elevado de intrusión en la vida del paciente, que interfiere con el desarrollo de su autonomía. Otro correlato de la EE lo constituyen las distintas atribuciones del origen de los trastornos esquizofrénicos: Así, en el caso de los familiares con un bajo nivel de EE una atribución de los síntomas a una enfermedad genuina se corresponde con un nivel superior de tolerancia tanto hacia los trastornos como hacia las dificultades de adaptación que el paciente experimenta a largo plazo.

En relación, igualmente, con la naturaleza de la EE, mencionemos, finalmente, los estudios sobre el análisis de la interacción familiar. Estos estudios han puesto de relieve correlatos conductuales de la EE congruentes con lo anteriormente expresado: un menor tiempo de verbalización de los sujetos esquizofrénicos en su interacción con familiares de alta $\mathrm{EE}$, mayor nivel de intrusividad y mayor frecuencia de críticas y de comportamientos que pueden considerarse intrusivos por parte de este tipo de familiares (Doane, West, Goldstein, Rodnick y Jones, 1981). A partir de estos estudios, incluso, se han desarrollado nuevos métodos de evaluación del clima familiar.

Estos distintos estilos de interacción, por otra parte, parecen diferenciar, no sólo a las familias de baja y alta EE, sino a distintos subgrupos déntro de las familias de alta EE. Así, se ha comprobado que la interacción directa con los pacientes de familiares con un alto nivel de criticismo (ya sea sólo o combinado con IEE) se caracteriza por una actitud igualmente crírica, mientras que los familiares con IEE presentan, más bien, una interacción de tipo intrúsivo (Miklowitz, Goldstein, Faloon y Doane, 1984).

En cuanto a la naturaleza de estas medidas, Greenley (1986) ha propuesto una interpretación alternativa. Este autor reinterpreta este concepto desde la perspectiva del control social y propone, en consecuencia, que lo que se está midiendo realmente son los intentos de los familiares por controlar, recurriendo a los mecanismos típicos de control interpersonal, si bien en forma muy intensa, las conductas desviadas de estos pacientes. Buscando evidencia que avalara su reformulación, este autor revisó los datos del trabajo inicial de Brown, Birley y Wing (1972) con objeto de comprobar si resultaban ciertas las predicciones que se derivaban de su teoría. En efecto, si su interpretación de la conducta de los familiares como una estrategia para hacer frente a la conducta desviada del paciente era correcta, podría comprobarse que a mayor grado de ansiedad y miedo que experimente una familia, más intensos serán sus esfuerzos para controlar la conducta del paciente. Adicionalmente, sin embargo, cabe pensar que esta relación vendrá modulada por la atribución que dichos familiares realicen de la causa de la conducta del paciente. En consecuencia, la relación antes apuntada sólo se observará cuando los familiares no atribuyan dicha conducta a enfermedad (considerándola, por ello, incontrolable así como eximiendo al paciente de toda responsabilidad sobre ella). En efecto, el análisis de los datos del estudio original corroboró las esperadas relaciones. 
Es interesante notar que esta interpretación permite explicar el beneficio clínico que se deriva de la explicación a los familiares del trastorno como una enfermedad (ver epígrafe 4), así como el valor no específico de estas medidas en el curso de los trastornos esquizof rénicos. Efectivamente, como veremos a continuación, el valor pronóstico de la EE que venimos comentando no se limita a estos trastornos, sino que es igualmente aplicable a otros.

En efecto, desde el trabajo de Vaughn y Leff (1976a) se estableció que el concepto de EE no tenía una utilidad específica sino que, tal vez introduciendo algunas modificaciones, podría ser aplicable a otros trastornos como los depresivos. Estos autores encontraron que, con pacientes depresivos, era necesario modificar los puntos de corte para obtener una predicción semejante a la lograda en esquizofrenia. Asimismo, comprobaron que una IEE se encontraba mucho más raramente, tal vez debido al hecho de que la mayor parte de estos pacientes estaban casados, por lo que esta medida apenas revestía interés para este grupo concreto de sujetos. Asimismo, se ha abordado el estudio de la posible utilidad de este concepto en el curso de otros trastornos como la anorexia (Szmukler, Eisler, Russell y Dare, 1985), el mantenimiento de la pérdida de peso (Fischmann-Havstad y Marston, 1984) o la epilepsia (Vaughn, comunicación personal). En todos estos ámbitos podemos decir, de un modo sucinto, que el concepto se ha demostrado útil. Aunque no se ha explorado, es evidente la urilidad que podría revestir en trastornos como determinados tipos de asma infantil.

Resulta por otra parte prioritario, tal como demuestra el proyecto emprendido por la OMS (Proyecto 5.2, citado por Kuipers, 1979), actualmente ya concluido y en vías de publicación, llevar a cabo estudios transculturales que pongan de relieve la utilidad de estas medidas en ámbitos culturales distintos. De otro modo dicho, factores como la cohesión familiar, la extensión de la familia o la amplitud de la red social, así como la tolerancia social hacia las conductas desviadas, pueden variar, naturalmente, de un contexto cultural a otro; en la medida en que éstas constituyen posibles variables moduladoras, pueden quedar con ello modificadas las relaciones entre EE y pronóstico a las que hemos aludido repetidamente. En los estudios llevados a cabo hasta el presente, sin embargo, este constructo ha demostrado su utilidad en dos culturas próximas, como la inglesa y norteamericana, así como entre hispanoparlantes residentes en los Estados Unidos. Los autores de este trabajo, por otra parte, están llevando a cabo un estudio en el ámbito español con objeto de estudiar la posible utilidad de este concepto en nuestro contexto cultural.

Señalemos, finalmente, que resulta curiosa la falta de vinculación de estos estudios con otros realizados en áreas de investigación más básica, como los de interacción o expresión emocional, según se desprende del análisis de los planteamientos teóricos en los que se basan los estudios de EE. Estos estudios han faltado incluso, hasta muy recientemente, en la exploración de las posibles relaciones con conceptos relativamente próximos e igualmente aplicables a los familiares de sujetos esquizofrénicos. Miklowitz, Strachan, Goldstein, Doane, et al. (1986) abordaron el estudio de la relación entre la EE y la desviación en la comunicación (DC). Ambos conceptos se refieren a dimensiones de la comunicación familiar, aunque, podríamos decir, a distintos niveles. Efectivamente, mientras la $\mathrm{EE}$ tendría más que ver con el contenido de las emociones que se manifiestan (en definitiva, se referiría a una disfunción en la comunicación emocional), la DC se refiere a aspectos formáles del lenguaje que los familiares utilizan 
para comunicarse. No obstante, en la medida en que ambas se han podido relacionar con el inicio de trastornos del espectro de la esquizofrenia y parecen tener un valor pronóstico considerable, es esperable que se presente algún tipo de solapamiento entre estas dos dimensiones. Los datos obtenidos por estos autores indican que los familiares de alta EE presentan niveles de CD igualmente superiores que los de baja EE. No obstante, se encontraron igualmente diferencias significativas en la $\mathrm{CD}$ entre los familiares de alta EE. En efecto, los familiares cuya çasificación como altos en EE se debía exclusivamente a un alto nivel de criticismo no diferían de los de baja EE en CD. Dentro de los familiares con IEE, por otra parte, el subgrupo que presenta Criticismo e IEE simultáneamente obtuvo las puntuaciones máximas en $\mathrm{CD}$. Esto puede, tal vez, interpretarse como reflejo de una actitud confusa o ambivalente en relación con el paciente. La asociación de este patrón de alta EE y CD puede implicar que estos pacientes tengan que hacer frente, junto a las dificultades asociadas a la convivencia con familiares con actitudes tan contrarias, a una comunicación particularmente difícil de decodificar y que aumentaría consiguientemente la confusión y la tensión. No obstante, no existen todavía datos que indiquen si esta asociación agrava de modo considerable el pronóstico de los pacientes que se ven sometidos a ella.

Como ya hemos indicado, uno de los últimos desarrollos en esta área es el de programas de intervención dirigidos a modificar estas conductas, y que revisaremos en el epígrafe siguiente.

\section{Implicaciones y desarrollos terapéuticos de los estudios de emoción expresada}

A lo largo de las páginas anteriores se ha puesto de manifiesto la fuer-. te asociación existente entre la EE y la recaída de los pacientes esquizofrénicos. Las actitudes familiares que componen el constructo de EE parecen constituir una fuente de estrés importante que al incidir en personas con un alto nivel de vulnerabilidad puede desencadenar la aparición de un episodio pṣicótico abierto. La posibilidad de delimitar un grupo de alto riesgo constituido por los pacientes esquizofrénicos que conviven con familiares de alta EE ha permitido diseñar estrategias terapéuticas que traten de prevenir la aparición de nuevas recaídas. Como hemos visto, este grupo de estudios constituyen la base empírica, a partir de la cual se han podido aislar distintos factores que desempeñan un papel importante en la aparición de recaídas. Entre éstos se incluyen las variables del ambiente familiar recogidas en la EE, el nivel de contacto, la ausencia de toma de medicación regular y la aparición de acontecimientos estresantes (Leff, Kuipers, Berkowitz, Vaughn y Sturgeon, 1983). Una vez conocida la participación de estos factores, estamos ante la posibilidad de modificar las variables de interés, diseñando intervenciones adecuadas. En efecto, se ha comprobado en varios estudios recientes que mediante la utilización de terapias psicosociales es posible disminuir las tasas de recaídas.

Los diferentes tipos de intervención desarrollados tienen una serie de puntos en común que pasamos a describir. En primer lugar, todos ellos han partido de los estudios de EE, constituyendo el grupo de alto riesgo, esquizofrénicos que conviven con familiares de alta EE; dentro de ellos, aquellos que están en alto contacto y que no toman medicación antipsicótica regularmente, constituyen, como sabemos, el de riesgo más elevado. En segundo lugar, todas las estrategias de intervención han sido evaluadas 
en forma controlada, lo que permite conocer de un modo objetivo su eficacia, la utilidad de sus componentes y su valor frente a otras formas de tratamiento. Señalemos, por último, la atención prestada desde esta óptica al aspecto puramente educativo del tratamiento. Aunque las distintas estrategias enfatizan en distinto grado este aspecto del tratamiento (ver Zipple y Spaniol, 1984), es notable en todas ellas la relevancia que adquiere la educación de los familiares en torno al conocimiento de la esquizofrenia, en todas sus facetas (etiología, sintomatología, curso y tratamiento). Esta característica supone una aportación nueva en la concepción del papel de los familiares en el tatamiento de la esquizofrenia. En efecto, frente a la postura más tradicional, en la que se venía cargándoles con un rótulo más o menos explícito de causantes (Kreisman y Joy, 1974), dsde esta nueva óptica se intenta eliminar los posibles sentimientos de culpa y vergüenza (Creer y Wing, 1974) que los familiares suelen padecer al tener un miembro esquizof rénico, para ser vistos como «réactores» ante esta circunstancia. Los familiares dejan de ser considerados como «agentes tóxicos» para pasar a jugar un papel de suma importancia en la recuperación del paciente. Este metamensaje está presente a lo largo de toda la intervención, y facilita que toda la familia se comprometa en el tratamiento y como consecuencia sea más sencillo conseguir los objetivos propuestos. El punto de partida de esta visión lo constituyen los trabajos sobre EE, que han puesto de relieve que no todos los familiares de los esquizofrénicos tienen un efecto perjudicial sobre ellos; por el contrario, aquellos que son considerados de baja EE parecen tener un efecto tranquilizante sobre sus familiares esquizofrénicos (Tarrier et al., 1979; Sturgeon et al., 1981, 1984), manteniendo una serie de actitudes que repercuten positivamente sobre el paciente (Vaughn y Leff, 1981). Por otra parte, la revisión minuciosa de aquellos estudios que han pretendido aislar alguna característica específica en las familias de esquizofrénicos, frente a las de sujetos normales, pone de manifiesto que no todas las familias de esquizofrénicos presentan anormalidades en sus estructuras o en sus pautas de comunicación o que dichas anomalías también pueden ser encontradas en las familias normales, no siendo, por tanto, exclusivas de las familias de esquizofrénicos (ver Hirsch y Leff, 1975).

Desde esta perspectiva, se han desarrollado dos grandes modelos de intervención, el primero de ellos llevado a cabo en Londres, por el equipo del Medical Research Council de la Unidad de Psiquiatría Social (Leff, Kuipers, Berkowitz, Eberlein-Vries y Sturgeon, 1982 y 1984) y el segundo, el modelo de Terapia Familiar Conductual, elaborado en el Centro de Estudios de la Esquizofrenia de Camarillo de la Universidad de California (Falloon, Boyd, McGill, Moss y Gilderman, 1982).

\subsection{La experiencia británica: el paquete de intervenciones sociales.}

Remitimos al lector al artículo de Leff et al., incluido en este mismo número, en el que queda ampliamente descrito el procedimiento y el tipo de intervenciones utilizadas por el grupo de investigadores británicos. En otra parte (ver Berkowitz, 1984; Berkowitz, Kuipers, Eberlein-Vries y Leff, 1981) los mismos autores ofrecen de forma más detallada los aspectos clínicos del paquete de intervenciones sociales desarrollado por ellos, compuesto por un programa educativo, un grupo de familiares y sesiones de rerapia familiar. El programa educativo se ha mostrado útil a la hora de incrementar el conocimiento y la comprensión de la esquizofrenia (Ber- 
kowitz et al., 1984). Asimismo, facilita el desarrollo de actitudes más optimistas y de la capacidad de percibir al paciente como una persona y no exclusivamente como alguien que padece un serio trastorno. Estos datos permiten concebir la educación familiar como una forma adecuada de ayuda, y que como mínimo, sobre la base de la opinión positiva expresada por los propios familiares, constituye un poderoso medio de conseguir su colaboración.

El segundo elemento del paquete, el grupo de familiares, se dirige a cubrir el déficit en apoyo emocional (Spaniol y Jung, 1984) que los propios familiares padecen. La oportunidad de pertenecer a un grupo de estas características permite reducir los sentimientos de aislamiento, ventilar sentimientos reprimidos respecto al paciente sin que éste se halle presente, y compararse con personas que sufren situaciones similares, con las que establecer un intercambio en el que puedan aprender o enseñar. La arención prestada a la comunicación dentro del grupo, y el enfoque de resolución de problemas sobre el que descansa el mismo, son dos importantes dimensiones en el logro de los objetivos perseguidos.

Finalmente, las sesiones de terapia familiar, que en palabras de Leff y Vaughn (1985) son «las más difíciles de describir en detalle» (pág. 128), constituyen posiblemente el aspecto menos elaborado del paquete de intervenciones, y cuya réplica presenta mayores dificultades.

En la traducción del artículo de 1982 de Leff y sus colaboradores podemos observar el éxito conseguido con este tipo de intervenciones en la reducción de la tasa de recaídas. Más recientemente, estos resultados logrados tras nueve meses de seguimiento han sido ampliados. Igualmente, tras un período de dos años (Leff, Kuipers, Berkowitz, Eberlein-Vries y Sturgeon, 1984; Leff, Kuipers, Berkowitz y Sturgeon, 1985), se mantienen los beneficios derivados de las intervenciones familiares destinadas a reducir el nivel de EE y el contacto con el paciente. Los pacientes que continuaron tomando la medicación de forma regular, redujeron sus recaídas en grañ medida. Sólo el 14 por 100 de los pacientes pertenecientes a familias experimentales en las que se redujo el nivel de EE y/o el contacto recayeron a lo largo de los dos años de seguimiento, en comparación con el 78 por 100 de recaídas de los pacientes controles con medicación regular $(\mathrm{P}=0,02)$.

Estos resultados vuelven a confirmar el papel causal de la EE en las recaídas, la utilidad terapéutica de las intervenciones sociales utilizadas y la necesidad imprescindible de tomar medicación regular, sobre todo en la medida en que el paciente conviva con familiares de alta EE.

A pesar de la importancia clínica que suponen estos descubrimientos, existen algunas limitaciones en los estudios citados que impiden esclarecer la fuente de eficacia. Dado que el tratamiento consistía en un paquete conjunto de intervenciones, no nos permite la evaluación aislada de cada uno de los elementos. Como Zipple y Spaniol (1984) señalan, este tipo de enfoque obtiene unos resultados similares a los de otro tipo de intervenciones más sencillas, pudiendo ser excesivos los costes temporales y económicos que implica. Estudios futuros que dispongan de una muestra suficientemente amplia deberían comparar la eficacia aislada de cada uno de los componentes del paquete. Mientras ello se lleva a cabo, el programa educativo diseñado parece ser útil en el cambio de atribuciones de las conductas problemáticas de los pacientes, el grupo de familiares para compartir las dificultades asociadas a ellas y aprender nuevas estrategias de enfrentamiento y las sesiones de terapia familiar, finalmente, para modificar el delicado tema de la separación. 
Desde el modelo de la vulnerabilidad (ver Zubin y Spring, 1977, y Vizcarro, en preparación), la reaparición o exacerbación de síntomas depende de la interacción que se establece entre la predisposición biológica del individuo, el nivel de estrés al que se ve sometido y su capacidad para enfrentarse ante él mismo. La investigación sobre $\mathrm{EE}$ ha hecho notar que la presencia de familiares altamente críticos o implicados con el paciente, constituye un estresor de gran magnitud y estabilidad. Por otro lado, no todos los familiares ejercen este efecto perturbador. Muy por el contrario, algunos disponen de mecanismos de enfrentamiento eficaces, resolviendo los problemas con calma y objetividad, evitando de este modo que las recaídas sean más frecuentes (Vaughn, 1977). La delimitación de una muestra de alto riesgo, constituida por los esquizofrénicos con familiares de alta EE, y la constatación de modalidades de enfrentamiento «sanas» en algunas familias de esquizofrénicos, han confluido en el desarrollo de un modelo conductual de terapia familiar, gestado. en el Centro de Investigación Clínica de Salud Mental de Camarillo de la Universidad de California (Faloon et al., 1982), a lo largo de más de diez años (Liberman, 1970).

Este modelo, que coincide con los enfoques tradicionales de tratamiento familiar en tomar la unidad familiar como foco de la intervención terapéutica, se distingue de ellos en considerar a la familia como unidad básica de salud (Falloon, 1985) y determinante crucial de la recuperación del paciente y no como el núcleo originario de la patología. Según esta concepción, muchos de los esfuerzos aparentemente negativos, llevados a cabo por los familiares, componen la mejor solución disponible en ese momento para ellos. En consonancia con esta visión, se presta especial atención a los recursos naturales de los que dispone la familia, raramente puestos. de relieve por otros autores, que han concentrado su interés en pautas de interacción patogénicas, y que en proporción son mucho menos frecuentes. En este sentido, la salud es entendida no sólo como ausencia de efectos negativos, tales como las recaídas, sino como el logro del máximo funcionamiento social competente por parte de todos los miembros de la familia. Con este propósito, el objeto principal del modelo es conseguir que la familia aprenda a resolver de forma efectiva los problemas cotidianos, para poder hacer frente al estrés asociado a la presencia de un familiar con esquizofrenia o al que puede desencadenarse por la ocurrẹncia de acontecimientos vitales comunes. Para ello, antes de intervenir directamente, se realiza un análisis conductual del sistema familiar que comprende las fases siguientes:

1. Evaluación individual de cada miembro de la familia para establecer los recursos, déficit, metas, expectativas, actitudes, sentimientos e intercambios con el resto de la familia.

2. Evaluación de las interrelaciones entre las diferentes metas y problemas de los distintos miembros, es decir, un análisis funcional del sistema familiar.

3. Observar los intentos familiares para resolver un problema, en el marco natural o a través de situaciones estandarizadas, para detectar los elementos eficaces y no eficaces.

4. Formulación, por parte del terapeuta familiar, de un plan de tratamiento para subsanar los principales déficits en la resolución de problemas de la familia. 
Esta fase de evaluación no se acaba con la recogida de datos para establecer una línea base, sino que, por el contrario, se continúa como toda evaluación conductual a lo largo de todo el tratamiento, con objeto de introducir las correcciones que se estimen necesarias. Tras el análisis conductual, se pone en marcha el programa de tratamiento que consta de diez sesiones semanales de dos horas de duración.

Los objetivos específicos que se pretenden conseguir son tres: aumentar los conocimientos sobre la esquizofrenia y su aceptación, mejorar la comunicación intrafamiliar y desarrollar estrategias eficaces de resolución de problemas. Como consecuencia del logro de estos objetivos, se consigue indirectamente reducir la tensión familiar y cambiar actitudes perjudiciales, como son un exceso de críticas o de implicación emocional. Esto, a su vez conlleva una mejoría en el estado clínico del paciente, verificable en su menor número de recaídas, así como un aumento en su calidad de vida y en la del resto de los miembros de la familia (Falloon y Pederson, 1985; Falloon et al., 1985).

Las sesiones se dividen en cuatro bloques: a) educación sobre esquizofrenia; $b$ ) entrenamiento en comunicación; $c$ ) entrenamiento en resolución de problemas, y $d$ ) planificación de problemas extrahospitalarios. Cada una de las sesiones se estructura sobre la enseñanza a la familia de la resolución eficaz de problemas, prestándose más atención al proceso que al contenido. La sesión comienza con una revisión de los progresos conseguidos, seguida del entrenamiento en la habilidad que corresponda, para acabar con un plan de generalización. El papel del terapeuta consiste principalmente en coordinar, guiar y asistir, aunque a veces, si las necesidades lo requieren, tenga que intervenir más activamente.

La educación sobre la esquizofrenia abarca las dos primeras sesiones. En la sesión inicial se escucha la experiencia de los familiares, explicándoseles a continuación, en lenguaje comprensible, lo que se conoce en torno al origen, los síntomas; el curso y el tratamiento de la esquizofrenia; finalmente, se les facilitan folletos para conseguir una mayor asimilación de la información. La segunda sesión versa sobre la medicación, exponiéndose las razones de su utilidad, los tipos de fármacos, los efectos perjudiciales del consumo de otras drogas, la importancia de la detección temprana de síntomas prodrómicos para evitar recaídas, los efectos secundarios y el valor de las dosis óptimas. Para evaluar la cantidad de información adquirida se administra un cuestionario antes y después de las sesiones.

El entrenamiento en comunicación (Liberman, Aitchinson y Falloon, 1979), introducido en el programa para disminuir la tensión en el clima familiar y para facilitar la resolución de problemas, se centra en cuatro tipos de comunicación: 1. Expresión de sentimientos y retroalimentación positiva. 2. Capacidad para hacer demandas en forma positiva. 3. Capacidad para escuchar activamente las necesidades de los otros. 4. Expresión de sentimientos y emociones negativos.

Cada sesión está dedicada a una sola habilidad de comunicación siguiendo el siguiente esquema (Snyder y Liberman, 1981):

1. Revisión de las habilidades aprendidas en la sesión anterior.

2. Explicación de las razones por las que es necesario aprender la habilidad escogida.

3. Instrucciones paso a paso de cómo adquirirla y utilizarla.

4. Modelado a través de role-playing.

5. Asignación de tareas para casa, con objeto de poner en práctica la habilidad y generalizarla. 
6. Entrega de resúmenes escritos en los que se explican todos'los pasos citados.

Además de este entrenamiento en comunicación, la familia recibe un entrenamiento en resolución de problemas, enseñándoseles un método estructurado compuesto por seis pasos que reproduce el modelo de Goldfried y D'Zurilla (1969):

1. Identificación del problema.

2. Generar soluciones alternativas.

3. Discusión de los pros y los contras de cada solución.

4. Elección de la(s) mejor(es) solución(es) posibles y reales.

5. Planificar cómo alcanzar la solución.

6. Revisión del proceso.

El resto de las sesiones se dedican a la resolución de problemas específicos siguiendo la estructura anterior. Por último, la sesión final se ocupa de la revisión de los avances, las dificultades que permanecen sin resolver y la planificación del futuro poshospitalario, determinando la frecuencia de los encuentros posteriores, así como la utilización de los recursos sociales comunitarios. La larga experiencia acumulada por los autores sobre el modelo les ha llevado a la elaboración de un manual, en el que queda descrito con todo detalle el procedimiento a seguir en cada una de las sesiones y que sirve de guía para la actuación del terapeuta (Falloon, Boyd y McGill, 1984).

\section{Resultados}

La validez de la terapia familiar conductual ha sido evaluada en un estudio controlado (Falloon et al., 1982, Falloon et al., 1984) que ha utilizado una muestra de 39 esquizofrénicos de alto contacto con sus familiares de alta EE. Tras la estabilización del episodio agudo y el establecimiento de la dosis óptima de medicación, los sujetos eran asignados a dos tipos de tratamiento: familiar e individual. El tratamiento familiar consistía en 23 sesiones de una hora y media de terapia familiar conductual en el hogar, a lo largo de nueve meses, siguiendo las orientaciones descritas (ver Falloon et al., 1981); además de dos sesiones de educación familiar. El tratamiento individual estaba compuesto por 25 sesiones de una hora, de terapia individual, en el centro clínico, a lo largo de nueve meses; posteriormente, una sesión mensual durante 24 meses con el objeto de mantener los logros conseguidos. Además, los familiares recibían apoyo cuando se consideraba oportuno, realizándose encuentros conjuntos con toda la familia u ofreciendo la asistencia a grupos multifamiliares.

Las variables dependientes para comprobar la eficacia de uno y otro tratamiento, han sido múltiples, incluyéndose no sólo las correspondientes a los pacientes, sino también las relacionadas con el resto de la familia. Los pacientes fueron evaluados con respecto a las exacerbaciones clínicas, la duración de las estancias en el hospital u otro tipo de instituciones después del alta y la regularidad de la toma de medicación (Falloon et al., 1985). En el resto de la familia se contempló el nivel de psicopatología, el ajuste social, la impresión subjetiva de malestar y la eficacia de los esfuerzos de enfrentamiento (Falloon y Pederson, 1985).

Los resultados de este estudio señalan que el enfoque de tratamiento familiar era superior al enfoque individual en la reducción de la morbili- 
dad clínica y social del paciente (Falloon et al., 1985) y que sus beneficios van más allá, mejorando también el ajuste de la unidad familiar (Falloon y Pederson, 1985). Las familias que recibían terapia familiar conductual in-

- formaban de que el trastorno del paciente producía un efecto menos disruptivo en la vida familiar, presentaban una reducción de su sintomatología física y psicológica, situándose en un rango normal, y disminuían su sensación subjetiva de malestar, en comparación con los que eran asignados al tratamiento individual. En cuanto a los pacientes se comprobó, en un seguimiento a los nueve meses, que aquellos que recibían terapia familiar sufrían menos exacerbaciones de sintomatología, tasas menores de psicopatología, menos ingresos en el hospital, tendían a disminuir sus síntomas negativos y a recibir dosis reducidas de neurolépticos, en comparación con los pacientes asignados a la otra condición de tratamiento. Al cabo de 24 meses se mantenía esta pauta de morbilidad reducida, sin que pudiera achacarse a diversos factores pronósticos, sesgos de los jueces, diferencias en la ocurrencia de acontecimientos vitales estresantes o efectividad de la farmacoterapia.

Los resultados conseguidos ponen de relieve la eficacia del modelo de terapia familiar conductual que, a través de la enseñanza efectiva de la resolución de problemas, consigue la disminución de la tensión en el ambiente familiar y aumenta la competencia de la unidad familiar como un todo, para hacer frente a diferentes tipos de dificultades. Como es evidente, el procedimiento para conseguir estos objetivos se diferencia del utilizado en el paquete de intervenciones sociales diseñado por Leff y sus colaboradores (Leff et al., 1982) en algunos aspectos. En primer lugar, sus objetivos son más amplios, tratando de aumentar el nivel de salud de toda la familia y no reduciéndose exclusivamente a la disminución de la EE y de la tasa de recaídas. En relación con los componentes específicos de la EE, parte del entrenamiento en comunicación se dirige a la expresión de los sentimientos negativos por considerar que pueden promover en ocasiones una resolución más efectiva de los problemas. No obstante, la observación conductual de la interacción familiar (Falloon y Pederson, 1985) puso de manifiesto que a los tres meses de la intervención familiar los comentarios críticos e intrusivos disminuían, por lo que este tipo de terapia puede considerarse útil en la reducción de algunos correlatos conductuales de la EE (Miklowitz et al., 1984). En segundo lugar, más allá de la reducción de la «temperatura emocional» de la familia (Anderson et al., 1980), el enfoque de la resolución efectiva de problemas permite que la unidad familiar entera, y no sólo el paciente, aumenten su calidad de vida y dispongan de recursos adecuados para enfrentar nuevas situaciones problemáticas, preservando su equilibrio. Seguramente, fijándonos en el nivel similar de éxitos conseguidos por ambos métodos, debamos pensar que comparten una serie de características comunes que constituyen el núcleo terapéutico eficaz, ya que es difícil pensar que la resolución eficaz de problemas pueda producirse sin un descenso de las emociones negativas o que el mismo no constituya por sí mismo una forma efectiva de resolver problemas.

Como conclusión a este apartado podemos decir que los programas de tratamiento descritos, así como otros de características similares (ver Goldstein et al., 1978, 1981; Anderson, 1983), han demostrado claramente su eficacia en la reducción de la morbilidad psiquiátrica de los pacientes esquizofrénicos, así como la gran importancia del clima emocional familiar, tanto en la producción de recaídas, como en la recuperación del paciente. A diferencia de otras intervenciones psicológicas de escasa eficacia, 
los tratamientos familiares para la esquizofrenia recientemente desarrollados ofrecen resultados esperanzadores. Sin lograr la "curación», meta irreal hoy por hoy, la combinación de la medicación neuroléptica con tratamientos psicosociales (Falloon y Liberman, 1983) resulta sinergética, disminuyendo en gran medida la posibilidad de que la vulnerabilidad a la esquizofrenia llegue a manifestarse en sintomatología activa. Es bien conocido que mediante la farmacoterapia es posible actuar sobre la sintomatología activa pero que, sin embargo, ésta por lo menos no es tan eficaz frente a la sintomatología residual o negativa, que por el contrario sí parece responder ante intervenciones psicosociales como las descritas, a través de las cuales el esquizofrénico puede aumentar su capacidad de enfrentamiento y por tanto su ajuste social y su calidad de vida.

Los procedimientos para alcanzar estos objetivos pueden ser distintos, como hemos visto, pero todas ellas comparten una serie de notas que posiblemente constituyan el fundamento de su éxito. Nos referimos al papel de colaboradores asignado a los familiares, estableciéndose una fuerte alianza terapéurica desde el principio a la información y apoyo proporcionados y a la creación de un clima altamente predecible y estructurado (Anderson, 1983). Las diferencias entre los distintos modelos se encuentran en el énfasis que conceden a tres aspectos que, sin embargo, todos contemplan: la educación en el conocimiento de los hechos básicos de los trastornos esquizofrénicos, el entrenamiento en habilidades concretas y el paoyo emocional (Spaniol y Jung, 1984). El modelo de Leff comprende todos ellos, mientras que el de Falloon atiende principalmente al entrenamiento en habilidades, recogiendo también el aspecto educativo.Gran parte del éxito alcanzado por los distintos enfoques se apoya probablemente en la disminución de los componentes negativos del ambiente emocional al que el paciente se ve sometido, y a que todos ellos abordan en alguna medida parte de las principàles necesidades expresadas por los familiares de esquizofrénicos: información, apoyo emocional y desarrollo de respuestas efectivas para manejar la esquizofrenia y los estresores a ella asociados (Spaniol, Jung, Zipple y Fitzgerald, 1985). A la espera de que nuevos estudios controlados clarifiquen la aportación específica de los elementos manejados, - por el momento disponemos de medios terapéuticos para aumentar la eficacia de los neurolépticos, mejorando el curso del trastorno y aliviando simultáneamente a los familiares del pesado lastre que deben soportar.

\section{Conclusiones}

En resumen, la revisión de los estudios sobre EE en familiares de sujetos esquizofrénicos pone de relieve la adecuación y enorme utilidad de estas medidas. El único obstáculo a la incorporación de esta metodología a la práctica clínica, a la que con seguridad puede enriquecer de un modo considerable, lo constituye su alto costo tanto en términos del entrenamiento previo que exige como de su aplicación propiamente dicha. La investigación futura puede poner de relieve correlatos de la misma que se presten a una evaluación más simple de la EE, así como que nos brinden una idea más precisa de lo que se plasma en estas medidas que hasta el momento se han retenido desde una base exclusivamente empírica. En este sentido, la profundización en cuestiones teóricas de esta naturaleza, conjuntamente con el mayor refinamiento metodológico, deben redundar en aportaciones sustanciales para la práctica clínica.

Asimismo, la investigación futura deberá adentrarse en la influencia 
de distintas variables moduladoras, así como en la aplicabilidad del concepto en distintos ámbitos culturales y, tal vez, áreas de la patología, tanto física como de la conducta.

Por el momento, sin embargo, y especialmente si analizamos los estudios llevados a cabo hasta.el presente sobre el valor pronóstico de estas medidas juntamente con los intentos de modificación de las pautas de interacción familiar, y el subsiguiente efecto positivo sobre el curso de estos trastornos, la conclusión es, ineludiblemente, que nos encontramos ante un concepto enormemente útil de cara a la práctica clínica.

\section{Referencias}

ANDERSON, C. M. «A psychoeducational model of family treatment for schizophrenia», en $\mathrm{H}$. Stierlin ét al. (eds.): Psychological interventions in schizophrenia. Heidelberg: Springer Verlag, 1983, págs. 227-235.

Anderson, C. M., OGARTY, G. E., y ReISS, D. J. «Family treatment of adult schizophrenic patients: A researchbased psycho-educational approach». Schizophrenia Bulletin, 1980, 6, 490-505.

Bateson, G.; JaCKSON, D.; HaLEY, L. y WEAKLAND, J. «Towards a theory of schizophrenia». Behavioral Science, $1956,1,251-264$

BERKowITZ, R. «Therapeutic intervention with schizophrenic patients and their families: a description of a clinical research project». Journal of Family Therapy, 1984, 6, 211-233.

Berkowitz, R.; Eberlein-VRIES, R.; KUIPERS, L., y LeFF, J. «Educating relatives about schizophrenia». Scbizophrenia Bulletin, 1984, 10, 418-429.

- «Lowering expressed emotion in relatives of schizophrenics», en M. S. Goldstein (ed.); New developments in interventions with families of scbizophrenics. Londres: Jossey-Bass, 1981.

Brown, G. W.; BIRLEY, J. L. T., y WING, J. K. «Influence of family life in the course of schizophrenic disorders: A replication». British Journal of Psychiatry, 1973, 121, 241-258.

Brown, G. W.; Monck, E. M.; Carstairs, G. M., y WING, J. K. «The influence of family life on the course of schizophrenic illness". British Journal of Preventative Social Medicine, 1962, 16, 55-68.

BROWN, G. W., y RUTTER, M. L. «The measurement of family activities and relationships». Human Relations, $1966,19,241-263$

CREER, C., y WING, J. «Living with a schizophrenic patient». British Journal of Hospital Medecine, $1975,14,73-82$.

DOANE, J. A.; West, K. L.; Goldstein, M. J.; RodniCK, E. H., y Jones, J. E. «Parental communication deviance and affective style: Predictors of subsequent schizophrenia spectrum disorders in vulnerable adolescents». Archives of Generab Psychiatry, 1981; 38, 679-685.

FALLOON, I. R. H. «Communication and problem solving skills training with relapsing schizophrenics and their families», en M. R. Lausky (ed.): Family therapy and major psychopatbology, Nueva York: Grune \& Stratton, 1981.

- «Behavioral family therapy: A problem-solving approach to family coping», en J. Leff y C. Vaughn (eds.): Expressed Emotion in families. Nueva York: The Guilford Press, 1985.

Falloon, I. R. H.; BoYd, J. L., y McGill, C. W. Family care of schizophrenics. Nueva York: The Guilford Press, 1984.

Falion, I. R. H., Boyd, J. L.; MCGill, C. W.; Razani, J; Moss, H. B., y Gilderman, A. M. «Family management in the prevention of exacerbations of schizophrenia: A controlled study». New England Journal of Medecine. 1982, 306, 1437-1440.

Falloon, I. R. H.; Boyd, J. F.; MCGill, C. W.; Williamson, M.; Razani, J.; Moss, H. B.; Gilderman, A. M., y SIMPSON, G. M.«Family management in the prevention of morbidity of schizoprhrenia. Clinical outcome of a two-year longitudinal study». Archives of General Psychiatry, 1985, 42, 887-896.

FalloON, I. R. H., y LibERMAN, R. P. «Interactions between drug and psychosocial therapy in schizophrenia». Schizophrenia Bulletin, 1983, 9, 543-554

FALLOON, I. R. H., y PEDERSON, J. «Family management in the prevention or morbidity of schizophrenia: The adjustment of the family unit» British Journal of Psychiatry, 1985, 147, 156-163.

Falloon, I. R. H.; Williamson, M.; Razani, J.; Moss, H. B.; Gilderman, A. M., y Simpson, G. M. «Family versus individual management in the prevention of morbidity of schizophrenia: I. Clinical outcome of a twoyear controlled study». Archives of General Psychiatry, 1985.

Fischmann.HavstaD, L., y MaRston, A. R. "Weight loss maintenance as an aspect of family emotion and process». British Journal of Clinical Psychology, 1984, 23, 265-271.

GOLDING, S. L.; BECKER, E.; SHERMAN, y RAPPAPORT, J. «The behaviour expectations scale. Assessment of interaction with the mentally ill». Journal of Consulting and Clinical Psychology, 1975, 43, 109-120.

GolDSTEIN, M. J.; KopEikIN, H. S. «Short and long-term effects of combining drug and family therapy», en M. J. Goldstein (ed.) New directions for mental health services: New developments in interventions with families of schizophrenics, 12. San Francisco: Jossey-Bass, 1981.

Goldstein, M. H.; Rodnick, E. H.; Evans, J. R.; MaY, P. R. A., y STeinberg, M. R. «Drug and family therapy in the aftercare of acute schizophrenics». Archives of. General Psychiatry, 1978, 35, 1169-1177.

GreEnLEY, J. R. «Social control and expressed emotion». Journal of Nervous and Mental Disease, 1986, 174, 24-30.

HIRSCH, S. R., y LEFF, J. P. Abnormalities in parents of schizopbrenics. Maudsley Monograph $\mathrm{N}^{2} 22$. Londres: Oxford University Press, 1975.

KUIPERS, L. «Expressed emotion: A review». British Journal of Social and Clinical Psychology, 1979, 18, 237-243. 
KREISMAN, D. E., y JoY, V. D. «Family response to the mental illness of a relative: A review of the literature». Schizophrenia Bulletin, 1974, 10, 34-57.

LEFF, J. P. «Family treatment of schizophrenia», en K. Grauville-Grosșman (ed.): Recent advances in clinical psychiatry, 5. Londres: Churchill Livinstone, 1985.

LefF, J. P.; KuIPERS, L.; Berkowitz, R.; Eberlein.VRIES, R., y StURGEON, D. «A controlled trial of social intervention in the families os schizophrenic patients». British Journal of Psychiatry, 1982, 141, 121-134.

LefF, J. P.; Kuipers, L.; Ber KowitZ, R.; Eberlein-VRies R. y StURGeon, D. "Psychosocial relevance and benefit of neuroleptic maintenance: Experience in the United Kingdom». Journal of Clinical Psychiatry, 1984, 45, $43-49$.

LeFF, J. P.; Kuipers, L.; Berkowtiz, R.; VAughn, C., y Sturgeon, D. «Life events, relatives expressed emotion and maintenance neuroleptics in schizophrenic relapsem. Psychological Medicine, 1983, 13, 799-806.

LEFF, J. P., y VAUGHN, C. E. "The interaction of life events and relatives expressed emotion in schizophrenia and depressive neurosis". British Journal of Psychiatry, 1980, 136, 146-153.

- «The role of maintenance therapy and relatives expressed emotion in relapse of schizophrenia: A two-year follow-up». British Journal of Psychiatry, 1981, 139, 102-104.

- Expressed emotion in families: Its significance for mental illness. Nueva York: The Guilford Press, 1985.

Liberman, R. P. «Behavioral approaches to family and couple therapy». American Journal of Orthopsychiatry, $1970,40,106-118$

Libermaí, R. P.; Aitchinson, R. A., y Falloon, I. R. H. Family therapy in schizopbrenia: syllabus for therapists. Mental Health Clinical Research Center for the Study of Schizophrenia. Camarillo, UCLA, 1979.

Lidz, T. «Schizophrenia and the family». Psychiatry, 1958, 21, 21-27.

MaY, R.A.; TUmA, A. H., y DiXON, W. J. «Schizophrenia: A follow up sutdy of the result of three forms of therapy». Archives of General Psychiatry, 1981, 38, 776-784.

Miklowitz, D. J.; Goldostein, M. H., y FALLOON, I. R. H. «Premorbid and symptomatic characteristics of schizophrenics from families with high and low levels of expressed emotion». Journal of Abnormal Psychology, $1983,92,359 \cdot 367$

Miklowitz, D. J.; Strachan, A. M.; Goldstein, M. J.; Donne, J. A.; Snyder, K. S.; Hogarty; G.E., y Falloon, I. R. H. «Expressed emotion and communication deviance in the families of schizophrenics». Journal of $A b$ normal Psychology, 1986, 95, 60-66.

Miklowitz, D. J.; Goldstein, M. J.; Falloon, I. R. H., y DoAne, J. A. «Interactional correlates of expressed emotion in the families fo schizophrenics». British Journal of Psychiatry, 1984, 144, 482-487.

RUTTER, M., y BROWN, G. W. "The reliability and validity of measures of family life and relationships in families containing a psychiatric patient». Social Psychiatry, 1966, 1, 38-53.

Szmukler, G. I.; EISLeR, I.; Russell, F. M., y DARE, C. "Anorexia nerviosa, parental "expressed emotion" and dropping out of tratment». British Journal of Psychiatry, 1985, 147, 265-271.

SPANIOL, L., y Jung, H. Coping strategies of family caregivers. Center for Rehabilitation Research and Training in Mental Health. Boston University, 1964.

SPANIOl, L.; Jung, H.; ZiPple, A. M., y FiTzGerald, S, Families as a central resource in the rebabilitation of the severely psycbiatrically disaabled: Report of a national survey. Center for Rehabilitation Research and Training in Mental Health. Boston University, 1984.

Sturgeon, D.; KuIPERS, L.; Berkowitz, R.; TURPIN, G., y LeFF, J. P. «Psychophysiological responses of schizophrenic patients to high and low expressed emotion relatives». British Journal of Psychiatry, 1981, 138, 40-45.

Sturgeon, D.; Turpin, G.; Kuipers, L.; Berkowitz, R., y LeFF, J. P. «Psychophysiological responses of schizophrenic patients to high and low expressed emotion relatives: A follow-up study". British Jourmal of Psychiatry, 1984, 145, 62-69.

TARRIER, N.; VAughn, C. E.; LAder, M. H., y LeFF, J. P. «Bodily reactions to people and events in schizophrenia». Archives of General Psychiatry, 1979, 36, 311-315.

VAUGHN, C. E. "Patterns of interactions in families of schizophrenics», en H. Katschuig (ed.) Schizophrenia: The other side. Viena: Urban y Schwarzenberg, 1977.

VAUGHN, C. E. y LEFF, J. P. "The influence of family and social factors on the course of psychiatric illness: A comparison of schizophrenic and depressed neurotic patients». British Journal of Psychiatry, 1976a, 129, $125-137$.

- The measurement of expressed emotion in the families of psychiatric patients. British Journal of Social and Clinical Psychology, 1976b, 15, 157-165.

- «Patterns of emotional response in relatives of schizophrenic patients». Schizophrenia Bulletin, 1981, 7, 43-44.

VAUGhn, C. E.; SNYder, K. S.; Jones, S.; Freeman, W. B., y Falloon, I. R. H. «Family factors in schizophrenic relapse. Replication in California of British research of expressed emotion». Archives of General Psychiatry. 1984 41, 1169-1177.

VIzCARro, C.; Botella, J, y Ruiz VARGas, J. M. Implicaciones del modelo de vulnerabilidad a la esquizofrenia para la prevención de la cronificación de estos trastomos. Comunicación presentada al I Congreso de Evaluación Psicológica. Madrid, Septiembre 1984.

WYNNE, L. C.; RYCKOFF, J.; DAY, J., y MIRSCH, S. «Pseudo-mutuality in the family relations of schizophrenics». Psychiatry, 1958, 21, 205-220.

ZIPPLE, A., y SPANIOL, L. Current educational and supportive models of family intervention: $A$ review and sug gestions for their use. Center for Rehabilitation Research and Training in Mental Health. Boston University, 1984.

ZUBIN, J., y SpRING, B. «Vulnerability: A new view of schizophrenia». Journal of Abnormal Psychology, 1977, $86,103+126$

ZÚbiN, J., y STEINHAUeR, S. «How to break the logham in schizophrenia. A look beyond genetics». Journal of Nervous and Mental Disease, 1981, 169, 477-492. 